This item was submitted to Loughborough's Research Repository by the author.

Items in Figshare are protected by copyright, with all rights reserved, unless otherwise indicated.

\title{
Effect of aggregation on viscosity of colloidal suspension
}

PLEASE CITE THE PUBLISHED VERSION

http://dx.doi.org/10.1134/S1061933X10050108

\section{PUBLISHER}

Spinger Verlag (@ Pleiades Publishing)

\section{VERSION}

AM (Accepted Manuscript)

\section{LICENCE}

CC BY-NC-ND 4.0

\section{REPOSITORY RECORD}

Kovalchuk, Nina, Victor Starov, and R.G. Holdich. 2010. "Effect of Aggregation on Viscosity of Colloidal Suspension". figshare. https://hdl.handle.net/2134/7016. 
This item was submitted to Loughborough's Institutional Repository (https://dspace.lboro.ac.uk/) by the author and is made available under the following Creative Commons Licence conditions.

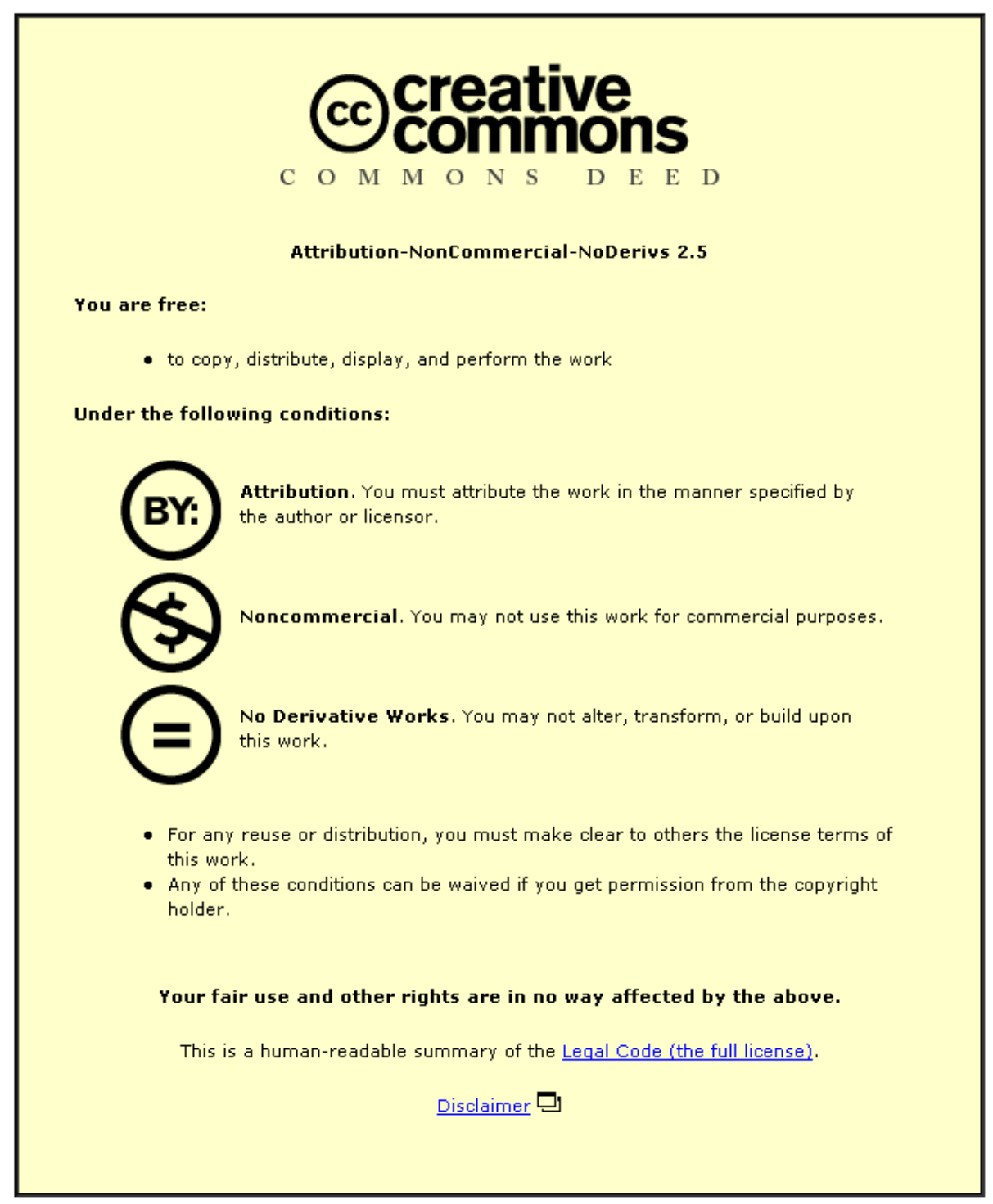

For the full text of this licence, please go to: http://creativecommons.org/licenses/by-nc-nd/2.5/ 


\title{
Effect of aggregation on viscosity of colloidal suspension
}

Nina Kovalchuk ${ }^{1,2}$, Victor Starov ${ }^{1 \mathrm{f}}$, Richard Holdich ${ }^{1}$

${ }^{1}$ Department of Chemical Engineering, Loughborough University, Loughborough, LE11 $3 T U(U K)$

${ }^{2}$ Institute of Biocolloid Chemistry, 03142 Kiev, Ukraine

\begin{abstract}
This experimental study of viscosity of colloidal suspensions was performed using a monodisperse polystyrene latex with particle diameter of $1.15 \mu \mathrm{m}$ and a pH dependent negative Zeta potential of up to $120 \mathrm{mV}$ in aqueous solutions. The range of electrostatic repulsion between the particles was controlled by varying the concentration of potassium chloride. Suspensions under investigation were either in a stable, coagulated, or gelated, state depending on the salt concentration. Shear thinning behaviour was observed for all the samples studied. The dependence of viscosity on shear rate imposed was found to depend substantially on the salt concentration.
\end{abstract}

${ }^{\text {f }}$ Corresponding author: V.M.Starov@lboro.ac.uk 


\section{Introduction}

Aggregation of suspensions is encountered very often in everyday life and it is a crucial step in a number of industrial processes. For example, in solid-liquid separation it enables a considerable improvement of the effectiveness of filtration, both increasing filter performance and decreasing the water content in the filter cake. However, at the same time, aggregation changes drastically the rheological properties of suspensions and complicates the transportation of suspensions. This example shows clearly the importance of an understanding how, and to what extent, the aggregation affects the rheology of suspensions.

It is well established that a colloidal suspension composed of non-interacting particles is a Newtonian liquid up to $20-25 \%$ solid volume fraction [1,2]. At small volume fraction of particles (several volume percent) hydrodynamic interactions between them can be neglected and suspension viscosity is described by the well known Einstein equation $[3,4]$

$\eta=\eta_{0}\left(1+\frac{5}{2} \varphi\right)$

where $\eta$ is the viscosity of the suspension, $\eta_{0}$ is the viscosity of the dispersion medium and $\varphi$ is the solid volume fraction.

At larger concentrations viscosity can be calculated using the equation proposed by Batchelor [5]:

$\eta=\eta_{0}\left(1+2.5 \varphi+6.2 \varphi^{2}\right)$

Other values for the coefficient of $\varphi^{2}$ have been reported [6], but according to [7] the applicability of equations taking into account only the second order terms on $\varphi$ is rather limited, as this allows only an estimate of the viscosity of suspensions with solid volume fractions up to $0.15-0.2$, with an error of $10 \%$ or higher.

At solid volume fractions above $25 \%$, even those composed by hard spheres (HS), i.e. particles with no colloidal interactions, demonstrate non-Newtonian behaviour with Newtonian plateaus at low and high shear rates connected by a shear thinning region [2,8-10]. In some cases a shear-thickening region follows the high shear rate plateau [2,8]. It is generally accepted, that formation of shear-induced structures is responsible for shear thinning in HS suspensions [11]. For example, the experimental study performed in [12] showed that formation of a sliding layer structure, due to shear, gave results 1.5 times lower in viscosity of the suspension than that of the disordered structure with the same solid volume fraction. 
Formation of structures in suspensions under flow takes place if a time scale associated with the imposed flow is small compared to the time scale of Brownian motion of particles, i.e. at large suspension Peclet numbers [11]:

$P e=\frac{\eta_{0} a^{3} \dot{\gamma}}{k T}>1$,

where $P e$ is the Peclet number, $a$ is the particle radius, $k$ is the Boltzmann constant, $T$ is temperature and $\dot{\gamma}$ is the imposed shear rate. The latter conclusion is in line with the results of numerical simulations [13], which have shown that the shear thinning at relatively small Peclet numbers (following the first Newtonian plateau) is the result of a decrease of the Brownian contribution to the viscosity.

A number of equations have been proposed to describe the dependence of low shear rate viscosity of concentrated suspensions on solid volume fraction (see, for example [14] and references inside) but one of the most often used is due to Quemada [15]:

$\eta=\eta_{0}\left(1-\frac{\varphi}{\varphi_{\max }}\right)^{-2}$,

and Dougherty-Krieger equation [16]:

$\eta=\eta_{0}\left(1-\frac{\varphi}{\varphi_{\max }}\right)^{-[\eta] \varphi_{\max }}$,

where $\varphi_{\max }$ is the maximum solid volume fraction corresponding to the viscosity divergence and $[\eta]$ is the intrinsic viscosity, both are fitting parameters. The fitting of available experimental data undertaken in [16] and consequent research has shown that Eq (5) describes not only the limiting low shear rate viscosity, but also the high shear rate viscosity and therefore any viscosity-solid volume fraction relation in the shear thinning region by appropriate choice of fitting parameters. For the low shear rate limit the value of $[\eta]$ should close to 2.5, then Eq. (5) coincides with Eq. (1) at $\varphi \rightarrow 0$ Obviously, $\varphi_{\max }$ increases with the increase of the shear rate imposed and for the case of high shear viscosity $\varphi_{\max }$ could be close to the close packing limit. The values of $\varphi_{\max }(\dot{\gamma} \rightarrow 0)=0.67$ and $\varphi_{\max }(\dot{\gamma} \rightarrow \infty)=0.78$ were found in [16] for aqueous suspensions of polystyrene latex whereas $\varphi_{\max }(\dot{\gamma} \rightarrow 0)=0.63$ and $\varphi_{\max }(\dot{\gamma} \rightarrow \infty)=0.71$ were found in $[17,18]$ for sterically stabilised silica spheres dispersed in cyclohexane.

If aggregates are formed in a suspension then the viscosity of the suspension increases due to an immobilisation of the liquid inside the aggregates and the latter 
results in an increase of the apparent solid volume fraction $[8,10,19]$. The increase of the viscosity of suspensions due to aggregation caused by an electrolyte addition was observed in [9]. Note, aggregated suspensions demonstrate non-Newtonian behaviour at much smaller volume fractions, for example, as low as 7\% in [20].

Direct consideration of the influence of aggregation on the viscosity of suspensions in the low shear limit when particles (aggregates) distribution can be considered as random was performed in [21-24] based on the Bruggeman's differential method for determination of effective properties of non-homogenous media [25]. It turned out that the dependence of the viscosity of a suspension on solid volume fraction is, in this case, described by an equation rather similar to the Dougherty-Krieger's Eq. (5):

$\eta=\eta_{0}\left(1-\frac{\varphi}{\bar{\varphi}_{\max }}\right)^{-2.5 \bar{A}}$,

but the physical meaning of the parameters is quite different: $\bar{\varphi}_{\max }$ in Eq. (6) is the averaged packing density of particles in the aggregates and $\bar{A}$ is the averaged relative friction coefficient of aggregates, i.e. the ratio of friction force exerted to the aggregate to the corresponding force exerted to the solid particle. Therefore, according to the Eq. (6) viscosity of a suspension should decrease if the clusters becomes more compact, which is in line with [8,10,19].

If shear is imposed on an aggregated suspension its viscosity changes due to: (i) change in the cluster structure (a compaction of clusters with an increase of the shear rate), Eq (6) and (ii) rearrangement of clusters under shear, Eq. (5). Both reasons (i) and (ii) results in shear thinning behaviour. Therefore, it could be expected that a decrease in the viscosity occurs essentially quicker for an aggregated suspension than for a non-aggregated one. Below, are the results of a comparative experimental study on the rheology of aggregated and non-aggregated suspensions which are presented to confirm the above conclusion.

\section{Experimental}

Aqueous polystyrene latex suspension composed of monodisperse particles with diameter $1.15 \mu \mathrm{m}$ was supplied by Micropore Technologies Ltd, Loughborough, UK. Suspension was concentrated up to about $43 \%$ solid volume fraction with a Baird \& Tatlock, Auto Bench Centrifuge Mark IV at $3600 \mathrm{rpm}$ and then treated in an 
ultrasonic bath to destroy possible aggregates. The absence of aggregates in the stock suspension was confirmed by both microscopic study (Leitz Ergolux and Leica DM RX microscopes) and by measurement of particle size distribution (volume based) by laser diffraction (Horiba LA-920). Particle size distribution in the stock suspension is given in Fig. 1.

The stock suspension was diluted to the desired solid volume fraction using a potassium chloride salt (Fluka, $>99.5 \%$ ) solution in ultrapure water to destabilise the suspension and to encourage aggregate formation. After dilution, the suspension was stirred gently and left for equilibration over 24 hours. The Microscopic study on aggregation of the suspension depended on the concentration of salt $(\mathrm{KCl})$, these tests were performed at a solid volume fraction 0.001 .

Zeta-potential of the suspension was measured using a Malvern Zetasizer $3000 \mathrm{HSA}$ in the presence of $\mathrm{KCl}$; pH values were adjusted with hydrochloric acid (Aldrich, $1.0000 \mathrm{~N}$ solution in water).

The measurement of viscosity was performed using an AR 1000-N rheometer (TA Instruments) employing double concentric cylinder geometry and maintaining a stepwise change (with a step of $0.5 \mathrm{~s}^{-1}$ ) for shear rate. The measuring cell was thermostated and kept at a temperature of $25^{\circ} \mathrm{C}$. The cell was filled with suspension using a Pasteur pipette with a very wide mouth, to prevent, as much as possible, destruction of clusters during the filling. Suspensions were equilibrated for $20 \mathrm{~s}$ before the measurements started.

The shear thinning behaviour of suspensions, due to breakage of clusters as well as rearrangement of particles under flow conditions, is under consideration below. A region of relatively low shear rates (below $100 \mathrm{~s}^{-1}$ ) was selected for investigation. Samples with a relatively low solid volume fraction are expected to possess low viscosity and, therefore, shear rates should be large enough to provide reliable data. Hence, lower values of shear rates were chosen using the known measurement results for pure water, which provide reasonably stable values of viscosity, close to the known viscosity of water of $1 \mathrm{mPa}$. The measured values of the viscosity of suspensions were normalised with respect to the measured viscosity of water. The presented experimental data are the average values from 3 distinct measurements. Standard deviation is shown below by error bars.

\section{Results and discussions}


Zeta-potential of polystyrene latex particles used in this study was measured at $0.01 \mathrm{M}$ of $\mathrm{KCl}$ (Fig. 2). Fig. 2 clearly shows that particles bear a negative charge and at $\mathrm{pH}>3$ suspensions are expected to be stable if the electrical double layer is not suppressed by electrolyte addition. This conclusion was confirmed by microscopic observations (Fig. 3a). By comparison, even at the small volume fraction employed in the microscopic study (0.001) noticeable aggregation was observed at $0.1 \mathrm{M}$ of $\mathrm{KCl}$ in suspension (Fig. 3b) and large aggregates appear at $0.25 \mathrm{M} \mathrm{KCl} \mathrm{(Fig.} \mathrm{3c).} \mathrm{This}$ microscopic study confirmed that all samples of suspension with $\mathrm{KCl}$ used in the rheological studies were well aggregated.

The dependence of the viscosity on the imposed shear rate for an electrostatically stabilised suspension (without salt addition) is shown in Fig. 4. At low volume fraction $\varphi=0.125$ the suspension behaves practically as a Newtonian liquid and its viscosity is close to that calculated according to the Batchelor equation (2) [5] (straight line in Fig. 4). At larger volume fractions essentially non-Newtonian (shear thinning) behaviour was observed. All three curves presented in Fig. 4 are reasonably well fitted by Dougherty-Krieger equation with maximum volume fraction increasing from $\varphi_{m}=0.60$ at $\dot{\gamma}=10 \mathrm{~s}^{-1}$ to $\varphi_{m}=0.65$ at $\dot{\gamma}=40 \mathrm{~s}^{-1}$ and with intrinsic viscosity decreasing from $[\eta]=3.3$ at $\dot{\gamma}=10 \mathrm{~s}^{-1}$ to $[\eta]=2.9$ at $\dot{\gamma}>40 \mathrm{~s}^{-1}$ (high shear plateau). As noted earlier in the Introduction, the generally accepted values of maximum volume fraction for low and high shear rate plateaus are higher. The smaller than expected values obtained in this study are most probably due to electrostatic repulsion between particles. If we accept the cited above values of $\varphi_{\max }(\dot{\gamma} \rightarrow 0)=0.63$ and $\varphi_{\max }(\dot{\gamma} \rightarrow \infty)=0.71$ for HS suspension, then corresponding effective solid volume fractions for suspensions presented in Fig. 4 are $\varphi_{e f}=0.136$ (curve 1), $\varphi_{e f}=0.35$ (curve 2) and $\varphi_{e f}=0.47$ (curve 3) and intrinsic viscosity decreases from $[\eta]=3.0$ at $\dot{\gamma}=10 \mathrm{~s}^{-1}$ to $[\eta]=2.65$ at $\dot{\gamma}>40 \mathrm{~s}^{-1}$.

Addition of salt causes substantial shrinkage of the electrical double layer around the particles and, therefore, a decrease in the range of the electrostatic repulsion. The suspension loses its stability resulting in the formation of aggregates. At a large enough salt concentration and solid volume fraction aggregates form a spanning network, i.e. the suspension transforms to a gel. In this study gelation was observed at solid volume fraction of 0.32 for the salt concentrations above $0.2 \mathrm{M}$ and at solid volume fraction 0.43 at $0.1 \mathrm{M} \mathrm{KCl}$. 
Performed experiments showed that the aggregation results in a considerable increase of the suspension viscosity. For the suspension of solid volume fraction 0.125, which was essentially a Newtonian liquid in the non-aggregated state (Figs. 4 and 5, curve 1), non-Newtonian shear thinning behaviour was observed when aggregated (Fig. 5, curves 2 and 3). Both curves corresponding to $\mathrm{KCl}$ concentration of 0.2 and $0.3 \mathrm{M}$ reach a high shear rate plateau at $\dot{\gamma}>55 \mathrm{~s}^{-1}$. That means that at this shear rate the shear induced macrostructure of the suspension, as well as internal cluster structure, reach their steady state.

When using HS approximation for aggregates and applying Eq. (5) using the above parameter values at the high shear limit, the effective solid volume fractions for suspensions, corresponding to curves 2 and 3 in Fig. 5, are $\varphi_{e f}=0.41$ (curve 2) and $\varphi_{e f}$ $=0.43$ (curve 3), i.e. they fall in between curves 2 and 3 in Fig. 4 . If the changes in the effective solid volume fraction due to changes of aggregates under shear are neglected, then the dependence of viscosity on the shear rate for these two suspensions could be approximated as presented by curves 2a and 3a in Fig. 5. Comparison of the theoretical curves with corresponding experimental data (curves 2 and 3 in Fig. 5) clearly show that effective solid volume fraction at low shear rates was essentially higher than at high shear rates causing much more rapid decrease of the viscosity than could be expected for HS suspensions.

An increase in the viscosity of suspensions by addition of the salt becomes even more pronounced at higher solid volume fractions (Fig. 6). Comparison of Figs. 4 and 6 shows that at a solid volume fraction $\varphi=0.32$ addition of $0.25 \mathrm{M} \mathrm{KCl}$ results in more than a 20 -fold increase of viscosity at low shear rates and about 10-fold increase at high shear rates. One can speculate that at larger solid volume fraction the distance between particles becomes smaller, therefore aggregates grow more rapidly. The latter results in their looser structure, i.e. the larger effective solid volume fraction.

Dependence of viscosity of a HS suspension on solid volume fraction over a wide range of shear rates can be described using Dougherty-Krieger Eq. (5) with fitting parameters $\varphi_{\max }$ and $[\eta]$ depending on the shear rate imposed. Dependence of viscosity of aggregated suspension on solid volume fraction at small shear rates (first Newtonian plateau for HS suspension, when parameters of Dougherty-Krieger equation remains constant) can be described by Eq. (6) again with parameters $\bar{\varphi}_{\max }$ and $\bar{A}$ depending on shear rate. At higher shear rates both processes - formation of 
shear induced macrostructure in suspension and rearrangement of aggregates contribute to the shear thinning of aggregated suspension. As to our knowledge, there is no equation proposed, which describes the dependence of viscosity of aggregated suspension on the solid volume fraction taking into account both mentioned above processes. This is a challenge for future work.

\section{Conclusions}

Experimental investigations of rheology of aggregated suspensions have shown that aggregation causes considerable increase in suspension viscosity especially at low shear rates. It results in non-Newtonian shear thinning behaviour for suspensions with relatively low solid volume fraction, which in non-aggregated state are Newtonian with a constant viscosity. In the shear thinning region, the viscosity of aggregated suspensions decreases much more rapidly due to contribution of two parallel processes: (i) rearrangement of particles inside the aggregates making aggregates more compact and (ii) formation of shear induced structures at a macro-level. The last mechanism is responsible for the shear thinning behaviour in non-aggregated suspensions.

\section{Acknowledgement}

The authors would like to acknowledge the support from The Engineering and Physical Sciences Research Council, UK (Grant EP/C528557/1) and EU funded MULTYFLOW project.

\section{References}

1. Maron S.H., Fok S.M. Rheology of synthetic latex. V. Flow behaviour of lowtemperature GR-S latex. J. Coll. Int. Sci., 1955, 10, 482-493.

2. Larson R.G. The structure and rheology of complex fluids., NY-Oxford, Oxford University press, 1999.

3. Einstein A. Eine neue bestimmung der Molekuldimension, Ann. Physik, 1906, 19, 289-306.

4. Einstein A. Berichtung zu meiner Arbeit: Eine neue bestimmung der Molekuldimension, Ann. Physik, 1911, 34, 591-592.

5. Batchelor G.K. The effect of Brownian motion on the bulk stress in a suspension of spherical particles, J. Fluid. Mech., 1977, 83, 97-117. 
6. Happel J., Brenner H. Low Reynolds number hydrodynamics with special applications to particulate media. Prentice Hall, 1965.

7. Thomas D.G., Transport characteristics of suspension: VIII. A note on the viscosity of Newtonian suspensions of uniform spherical particles, J. Colloid Sci., 1965, 20, 267-277.

8. Barnes H.A., Hutton J.F., Walters K. An introduction to rheology, Elsevier, Amsterdam, 1989.

9. Krieger I.M. Rheology of monodisperse lattices, Adv. Coll. Int. Sci. 1972, 3, 111-136.

10. Quemada D., Berli C., Energy of interaction in colloids and its application in rheological modelling, $A d v$. Coll. Int. Sci., 2002, 98, 51-85.

11. Vermant J., Solomon M.J. Flow-induced structure in colloidal suspensions, J. Phys.: Condens. Matter, 2005, 17, R187-R216.

12. Wu Y. L., Derks D., van Blaaderen A., Imhof A. Melting and crystallization of colloidal hard-sphere suspensions under shear, PNAS, 2009, 106, 1056410569.

13. Bossis G., Brady J.F. The rheology of Brownian suspensions, J. Phys. Chem. 1989, 91, 1866-1874.

14. Zholkovskiy E.K., Adeyinka O.B., Masliyah J.H. Spherical cell approach for the effective viscosity of suspensions, J. Phys. Chem. B, 2006, 110, 1972619734.

15. Quemada D. Rheology of concentrated dispersed systems and minimum energy dissipation principle. I. Viscosity-concentration relationship, Rheol. Acta, 1977, 16, 82-94.

16. Krieger I.M., Dougherty T.J., A mechanism for non-Newtonian flow in suspensions of rigid spheres, Trans. Soc. Rheol. 1959, 3, 137-152.

17. de Kruif C.G., van Iersel E.M.F, Vrij A., Russel W.B. Hard sphere colloidal dispersions: viscosity as a function of shear rate and volume fraction, J Chem. Phys., 1985, 83, 4717-4725.

18. Jones D.A.R., Leary B., Boger D.V. The rheology of a concentrated colloidal suspension of hard spheres, J. Coll. Int. Sci., 1991, 147, 479-495.

19. Perez M., Barbe J.C., Neda Z., Brechet Y., Salvo L., Computer simulation of the microstructure and rheology of semi-solid alloys under shear, Acta mater., 2000, 48, 3773-3782. 
20. Friend J.P., Hunter R.J. Plastic flow behaviour of coagulated suspensions treated as a repeptisation phenomenon, J. Coll. Int. Sci., 1971, 37, 548-556.

21. Zhdanov V.G., Starov V.M., Determination of the effective viscosity of concentrated suspensions, Colloid J., 1998, 60, 771-774.

22. Starov V., Zhdanov V., Effective viscosity and permeability of porous medium, Coll. Surf. A, 2001, 192, 363-375.

23. Starov V., Zhdanov V., Meireles M., Molle C., Viscosity of concentrated suspensions: influence of cluster formation, Adv. Coll. Int. Sci., 2002, 96, 279293.

24. Starov V.M., Zhdanov V.G., Effective properties of suspensions/emulsions, porous and composite materials, Adv. Coll. Int. Sci., 2008, 137, 2-19.

25. Bruggeman, D.A.G., Berechnung verschiedener physikalisher Konstanten von heterogenen Substanzen. I. Dielektrizitatskonstanten und Leitfahigkeiten der Mischkorper aus isotropen Substanzen, Ann. Phys., 1935, vol. 24, p. 636-664.

\section{Figure legends}

Fig. 1. Particles size distribution in the stock suspension.

Fig. 2. Dependence of zeta-potential of polystyrene latex particles on $\mathrm{pH}$ of dispersion medium at $10^{-2} \mathrm{M} \mathrm{KCl}$.

Fig. 3. Aggregation in polystyrene latex suspension depending on salt concentration: a - 0.001 M KCl; b - 0.1 M KCl; c - 0.25 M KCl. Solid volume fraction 0.001.

Fig. 4. Dependence of viscosity of electrostatically stabilised suspension of polystyrene latex on the shear rate imposed. Solid volume fraction: $1-0.125,2-$ $0.32,3-0.43$

Fig. 5. Dependence of suspension viscosity on shear rate for various salt concentrations: 1 - without $\mathrm{KCl}, 2-0.2 \mathrm{M}, 3-0.3 \mathrm{M}$. Solid volume fraction 0.125. Compact lines 2a and 3a - corresponding fitting according to the Dougherty-Krieger equation (see text for details). 
Fig. 6. Dependence of suspension viscosity on shear rate at salt concentration $0.25 \mathrm{M}$ and solid volume fraction $1-0.18,2-0.32$. 


\section{Figures}

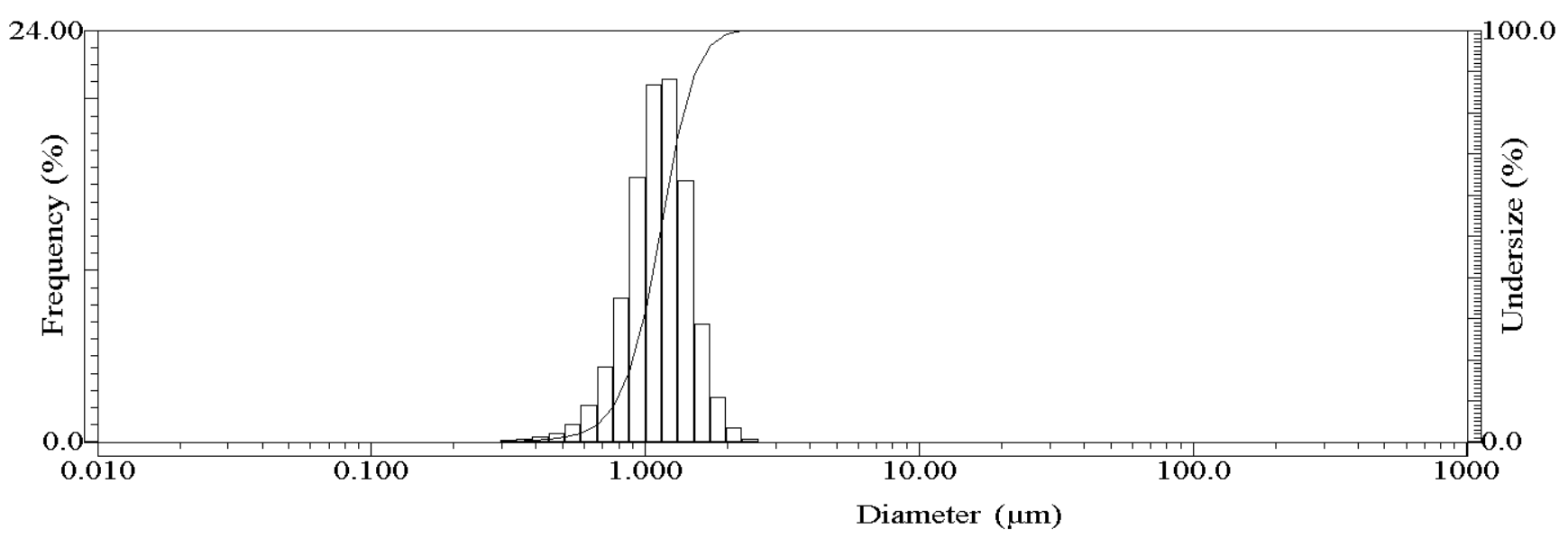

Fig. 1. Particles size distribution in the stock suspension.

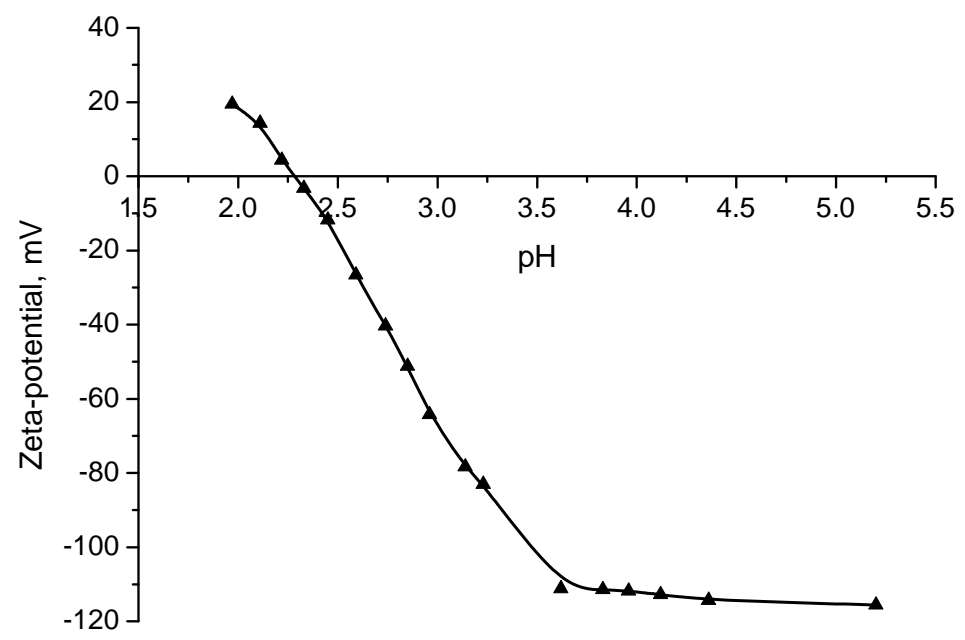

Fig. 2. Dependence of zeta-potential of polystyrene latex particles on $\mathrm{pH}$ of dispersion medium at $10^{-2} \mathrm{M} \mathrm{KCl}$. 


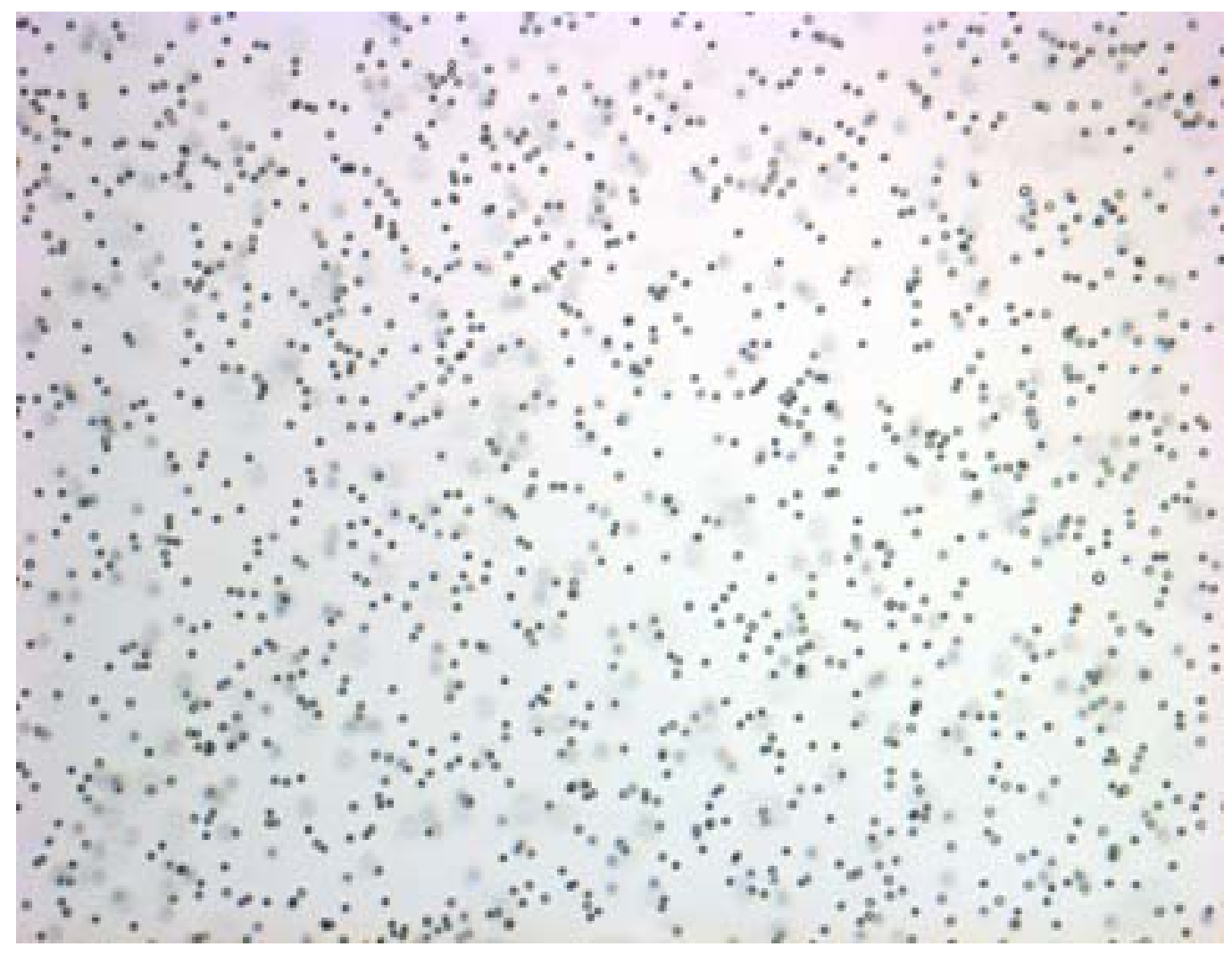

Fig. 3a. Aggregation in polystyrene latex suspension at $\mathrm{KCl}$ concentration 0.001 M. Solid volume fraction $0.1 \%$. 


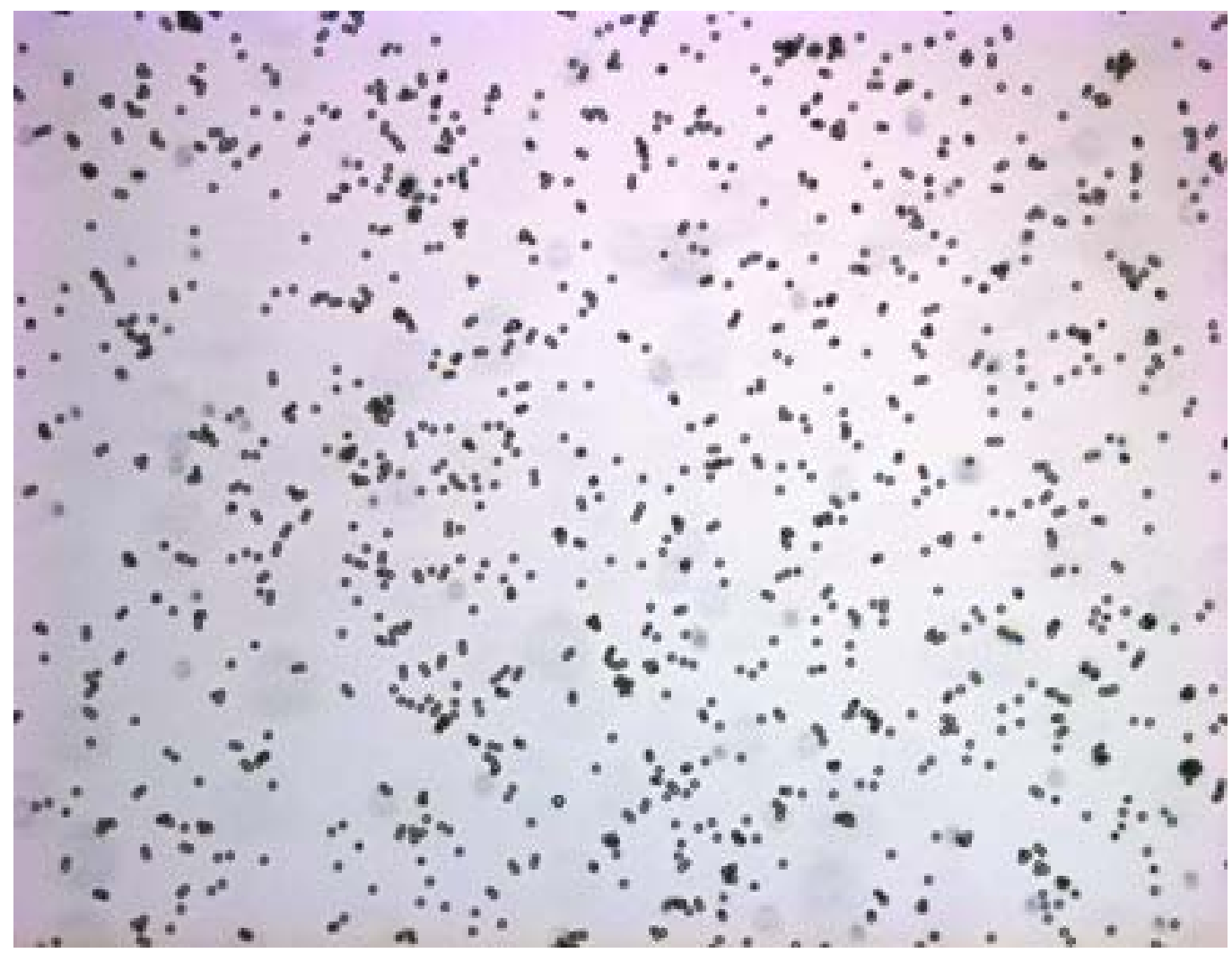

Fig. 3b. Aggregation in polystyrene latex suspension at $\mathrm{KCl}$ concentration $0.1 \mathrm{M}$. Solid volume fraction $0.1 \%$. 


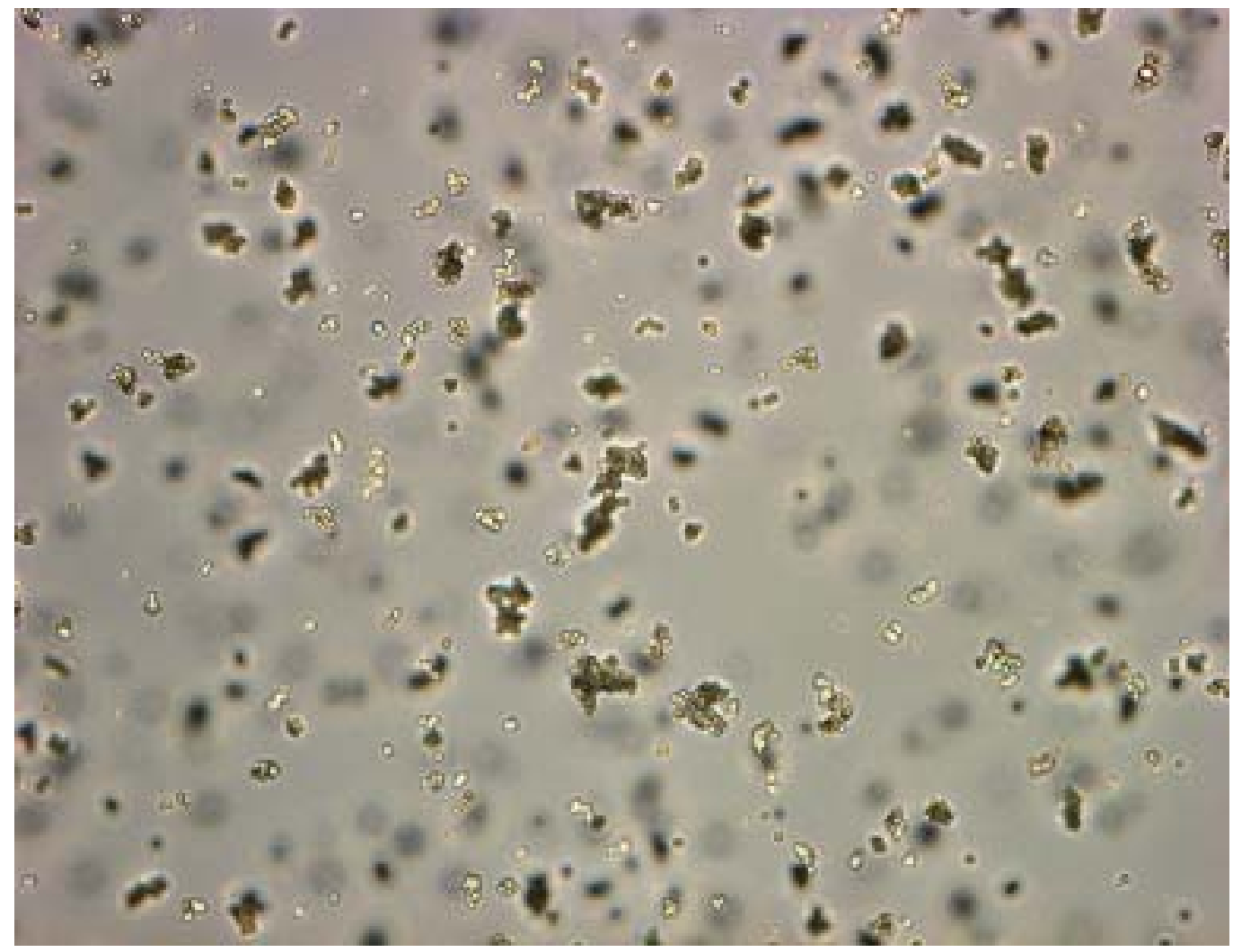

Fig. 3c. Aggregation in polystyrene latex suspension at $\mathrm{KCl}$ concentration $0.25 \mathrm{M}$. Solid volume fraction $0.1 \%$. 


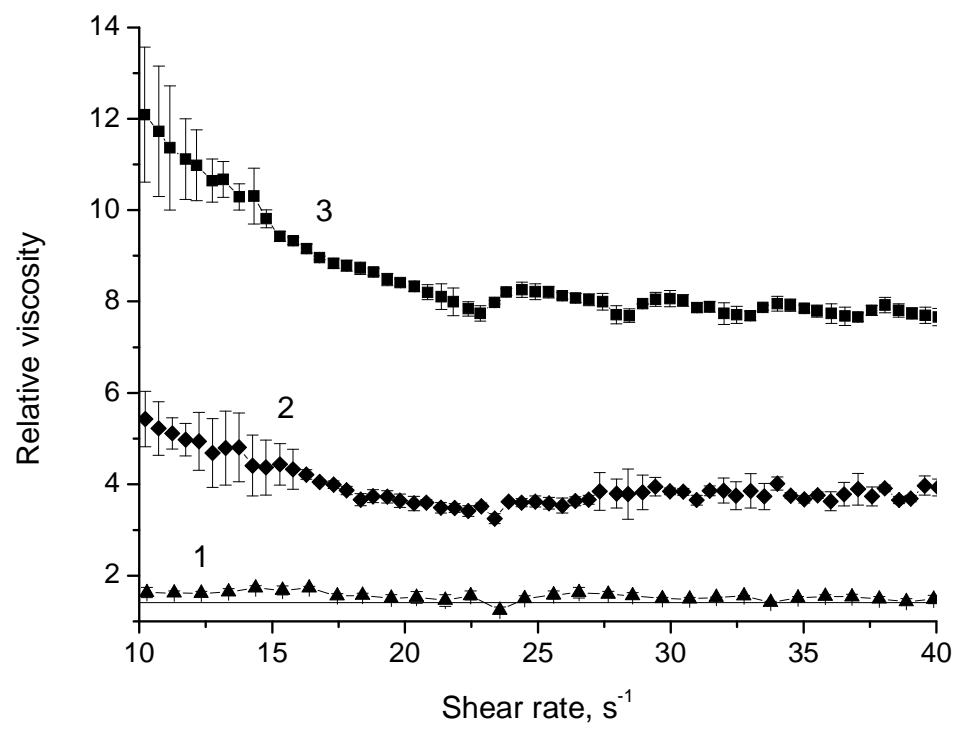

Fig. 4. Dependence of viscosity of electrostatically stabilised suspension of polystyrene latex on the shear rate imposed. Solid volume fraction: $1-0.125,2-$ $0.32,3-0.43$.

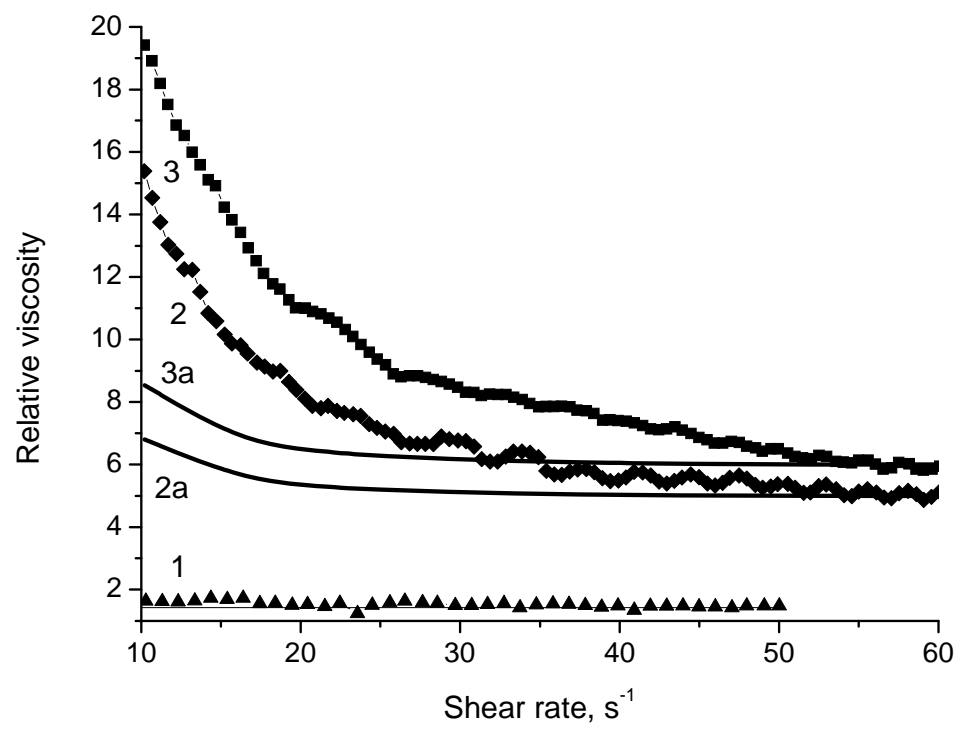

Fig. 5. Dependence of suspension viscosity on shear rate for various salt concentrations: 1 - without $\mathrm{KCl}, 2-0.2 \mathrm{M}, 3-0.3 \mathrm{M}$. Solid volume fraction 0.125. Compact lines 2a and 3a - corresponding fitting according to the Dougherty-Krieger equation (see text for details). 


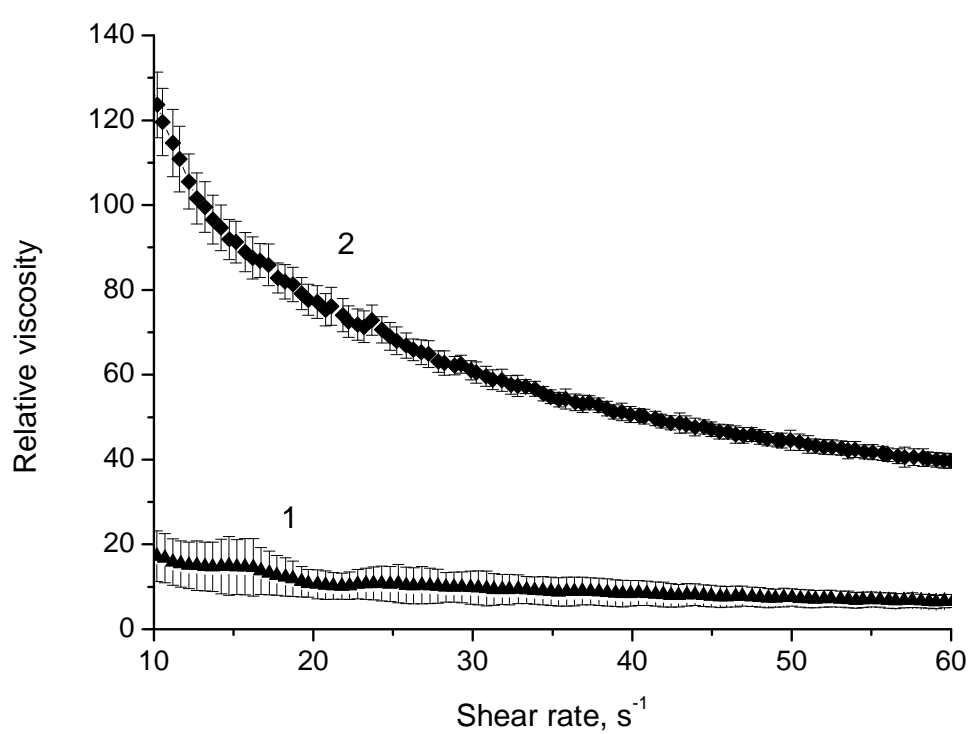

Fig. 6. Dependence of suspension viscosity on shear rate at salt concentration $0.25 \mathrm{M}$ and solid volume fraction $1-0.18,2-0.32$. 